\title{
La précaution « des » nanotechnologies
}

Une limitation et un contrôle des opérations du système par le monde vécu

Patrick Chaskiel

\section{(2) OpenEdition}

12 Journals

Édition électronique

URL : http://journals.openedition.org/communicationorganisation/4459

DOI : 10.4000/communicationorganisation.4459

ISSN : $1775-3546$

Éditeur

Presses universitaires de Bordeaux

\section{Édition imprimée}

Date de publication : 1 juin 2014

Pagination : 19-32

ISBN : 978-2-86781-904-9

ISSN : $1168-5549$

\section{Référence électronique}

Patrick Chaskiel, «La précaution « des » nanotechnologies », Communication et organisation [En ligne], 45 | 2014, mis en ligne le 01 juin 2017, consulté le 19 avril 2019. URL : http://journals.openedition.org/ communicationorganisation/4459; DOI : 10.4000/communicationorganisation.4459 


\title{
La précaution « des » nanotechnologies Une limitation et un contrôle des opérations du système par le monde vécu
}

\author{
Potrick Choskiel'
}

\section{Introduction}

De la théorie habermassienne, il a souvent été retenu, y compris par - le jeune - Habermas lui-même, l'idée d'une colonisation du monde vécu par le système pour caractériser les rapports entre ces deux sphères de la société, définies par les deux types d'opérations sociales que sont la régulation et la communication ${ }^{2}$. Habermas entendait par là que l'espace public bourgeois s'est étiolé sous l'effet d'une commercialisation extensive des activités sociales. Cette position, on le sait, s'inscrit (encore) largement dans une perspective francfortienne, pour partie issue de la théorie de la rationalisation bureaucratique (à travers le droit) et monétaire (à travers le calcul économique) de Weber.

Dans cette optique, les limites à l'expansion du système semblent avoir, sinon totalement disparu, tout au moins progressivement fondu dans les théories de la domination. Dans ce cas, les revendications des dominés sont considérées comme étant absorbées, voire retournées par le système, qu'il s'agisse des revendications salariales (qui sont susceptibles de faire mieux fonctionner l'économie à travers la consommation de masse) ou écologiques (la commercialisation croissante de produits « bio »).

Cette insistance mise sur la domination peut donc trouver un ancrage concret : la marchandisation mondialisée (la globalisation) ou le contrôle «numérique» des citoyens par les États (et leurs agences de renseignements), par exemple, tendent à moderniser cette version des rapports colonisateurs du système au monde vécu.

1 Patrick Chaskiel est professeur de Sciences de l'Information et de la Communication à l'Université Paul Sabatier-Toulouse III. Il est chercheur au CERTOP (Centre d'Études et de Recherches Travail, Organisation, Pouvoir), UMR 5044, CNRS, UJ2, UPS. Il est animateur de la thématique « Environnements, Risques, Vulnérabilités » au sein de la MSHS-Toulouse ; patrick.chaskiel@univ-tlse3.fr

2 Jürgen HABERMAS, L'espace public. Archéologie de la publicité comme dimension constitutive de la société bourgeoise, Paris, Payot, 1978. 
Une telle théorisation, critique et compréhensive, a certes pour avantage de fournir une version " académiquement correcte » de la critique sociale en raison de son caractère essentiellement analytique ${ }^{3}$, pouvant même prendre une allure statistique ${ }^{4}$. Néanmoins, parce que critique d'un bout à l'autre de la chaîne d'analyse, elle a pour effet de laisser de côté la question du potentiel d'émancipation auquel renvoie le monde vécu, et plus particulièrement l'espace public.

Cette réduction de l'analyse critique à la seule force de la domination s'écarte d'une théorisation de l'émancipation, d'ailleurs présente dans l'École de Francfort, mais qui ne l'a pas transformée en une pensée pratique : la formation d'un « homme nouveau » (Marcuse) ne saurait tenir lieu de conception opératoire d'une politique de l'émancipation.

La théorie analytique de la domination se détache ainsi d'une tradition dans laquelle description et compréhension de la domination ne peuvent être séparées d'une perspective normative d'émancipation. Cette dernière a certes quelques racines dans la posture hégélienne et le dépassement des extrêmes de la société civile-bourgeoise par l'État, même si domination et émancipation ne sont pas, ici, des concepts. Elle a été significativement transformée par Marx dont les textes politiques, plus que théoriques, ont esquissé ce que pourrait être une politique communiste, c'est-à-dire la possibilité d'une émancipation du prolétariat. Elle a encore, et surtout, fait l'objet d'une interprétation formalisée par Habermas, construisant une théorie de la démocratie en partant de la puissance attribuée à l'agir communicationnel (Habermas 1997).

\section{La thématique publique des risques technologiques}

Sans entrer dans la question procédurale envisagée par Habermas, notamment à travers l'usage du medium du droit, ce texte se propose de valoriser la notion de puissance communicationnelle, plutôt que " pouvoir communicationnel $»^{5}$ comme expression de la capacité du monde vécu, de l'espace public plus effectivement, à limiter et contrôler le fonctionnement du système.

Dans cette optique, on peut montrer que l'espace public, loin d'être marginalisé, constitue le foyer de la contestation sociale mais aussi l'espace de formation d'exigences vis-à-vis du système, ainsi que le suggère - et le préconise - d'ailleurs le « Habermas de la maturité ».

Par espace public, on entendra, de manière stricte, l'espace de déploiement de l'agir communicationnel. En d'autres termes, l'espace public opère comme un processus de filtrage des discussions sur la base d'un critère général

3 Parmi d'autres références : Pierre BOURDIEU, La distinction. Critique sociale du jugement, Paris, éd. de Minuit, 1979. Également : Luc BOLTANSKI et Eve CHIAPELLO, Le nouvel esprit du capitalisme, Paris, éd. Gallimard, 1999.

4 On pense évidemment au recours, sans doute provocateur, à l'analyse factorielle par Bourdieu.

5 Jürgen HABERMAS, Droit et démocratie. Entre faits et normes, Paris, Gallimard, 1997.

6 Ce qui est clairement indiqué à travers la notion de " démocratie délibérative ", Droit et Démocratie. op. cit. 
d'accessibilité, d'égalité et, particulièrement, de solidarité. Ce dernier aspect est académiquement sous-estimé au profit du premier, ce qui aboutit le plus souvent à l'idée, peu sophistiquée, que l'espace public ne serait qu'un espace " ouvert à tous ». Or, du point de vue de la théorie habermassienne, l'espace public se présente fondamentalement comme la sphère de formation d'exigences universalisables, c'est-à-dire détachées, indépendantes, des calculs monétaires et politiques (électoraux notamment).

Le système doit sans cesse répondre à ces exigences, parce que les décisions fonctionnelles ne peuvent durablement s'abstraire de légitimité. Même si ce besoin est plus fort pour le système politique (et son code électoral de fonctionnement fondé sur la relation entre majorité et opposition ${ }^{7}$ ) que pour le système économique, ce dernier en dépend de plus en plus face à des menaces de boycott de produits écologiquement ou éthiquement jugés problématiques. On désignera la formation de telles exigences universalisables et leur apposition au système comme un processus de «thématisation publique ».

En formant des exigences sur lesquelles le système n'a pas de prise hiérarchique ou monétaire, l'agir communicationnel a entraîné une complexification, donc un durcissement, de l'épreuve de légitimation des décisions fonctionnelles, politico-bureaucratiques et économiques.

La complexité envisagée ici ne relève pas du fonctionnement systémique lui-même contrairement à ce qu'envisage constamment Luhmann à travers l'unité de la variété des opérations du système ${ }^{8}$. Elle n'est pas plus une « irritation » du système par des opérations issues de son environnement. De telles conceptions ${ }^{9}$ tendent à faire du système l'élément central de la société, alors que la thèse défendue ici privilégie la confrontation entre le monde vécu et le système comme ressort social fondamental.

La thématisation publique des risques environnement-santé apparaît alors comme significative de la constitution des exigences universalisables. On la formalisera comme étant l'expression de la tension entre exigences écologiques (protection de l'environnement et de la santé) et opérations fonctionnelles (monétaires et bureaucratiques). Dès lors, on peut mettre en évidence le poids progressivement acquis par l'espace public dans sa confrontation avec le système, donc l'affaiblissement relatif de ce dernier dans son aptitude à maîtriser et orienter ses propres conditions d'expansion.

Une expression maturée de cette tendance est la mise en thème public des « nanotechnologies ». Cette thématisation prend la forme d'une remise en cause du bien-fondé de l'expansion des technologies de manipulation de la matière et de matériaux à l'échelle nanométrique. À cette échelle émergent des propriétés physico-chimiques « extraordinaires ", par exemple : résistance mécanique fortement accrue, conductivité électrique largement améliorée,

7 Niklas LUHMANN, Ecological Communication, Chicago, The University of Chicago Press, 1986.

8 Niklas LUHMANN, Theory of Society, volume 1, Stanford, Standford University Press, 2012, p. 78 et suiv.

9 Présentes dans les recherches académiques tournées vers les « organisations » ou les " politiques publiques ». 
auto-nettoyage ou effets bactéricides, par exemple ${ }^{10}$. La promotion des nanotechnologies constitue le levier d'une industrialisation centrée sur la conception et production de produits à haute valeur ajoutée ${ }^{11}$ plutôt que sur une production de masse fondée sur le principe de l'économie d'échelle, couramment incarnée par la notion de fordisme et son modèle de la production de masse.

Dans une histoire de la thématique publique des risques marquée par une accumulation de thèmes universalisables et singuliers (nucléaire, pollutions, amiante, OGM, ...), celui des nanotechnologies révèle, spécifiquement, une tension entre les problèmes d'applicabilité (techno-industrielle) et ceux d'implicabilité (sociétale) $)^{12}$.

L'applicabilité relève d'une stratégie, multiforme : scientifique, technologique et productive, supposant une décision concernant des projets technoindustriels ; l'implicabilité renvoie à des effets susceptibles d'être discutés en termes d'exigences universalisables, c'est-à-dire aussi à une possible mise en cause de la raison d'être de produits en raison de leurs effets écologiques potentiellement négatifs.

Sur cette base théorique, il est possible de montrer comment l'implicabilité pèse effectivement sur le développement des applications technologiques et industrielles ${ }^{13}$.

\section{De l'applicabilité des nanotechnologies ...}

En - et de - quoi les nanotechnologies figurent-elles une nouveauté ? Cette question peut paraître saugrenue tant s'est installée l'idée ordinaire que les nanotechnologies représentent une révolution scientifique et technologique. Pourtant, cette assertion ne va pas de soi, et on est conduit à prendre à contrepied une tendance institutionnelle, née aux États-Unis, puis mondialement déployée, y compris dans des pays sans grande tradition technoscientifique.

En pratique, la National Nanotechnology Initiative ${ }^{14}$ en coalisant - et en donnant du sens à - un ensemble d'opérations scientifiques dispersées, représente le point de départ d'une politique ${ }^{15}$ de financement, qui ne s'ancre

10 Dominique VINCK, Nano-technologies, Paris, Le Cavalier bleu, 2009.

11 Pour la Commission Européenne, les nanomatériaux et la nanoélectronique figurent parmi les « technologies génériques clés » de la décennie 2010. Voir également : "Le déploiement industriel des nanotechnologies et de la biologie de synthèse sur les territoires, précurseur des manufactures du futur ", rapport au gouvernement, n $13-1207$ DEF/CGARM/SG, décembre 2013.

12 On étendra par là la description menée par Christopher M. KELTY, « Beyond Implications and Applications: the Story of 'Safety by Design'», NanoEthics 3, no. 2, 2009, p. 79-96.

13 Les résultats exposés se situent dans le cadre d'une opération de recherche contractuelle : " Mobirisques ", financée par l'Anses (Agence Nationale de sécurité sanitaire, de l'alimentation, de l'environnement et du travail). 14 Mihail C. ROCO, NNI: From Vision to the Implementation. Brief presentation, November 2000, https:// www.nsf.gov/crssprgm/nano/reports/roco_vision.jsp.

15 Un temps qualifiée de « nanomania » par des chercheurs (entretiens). 
aucunement dans une dynamique scientifique, technologique ou industrielle, unifiée ${ }^{16}$.

Les nanotechnologies des années 2000 ne renvoient, en effet, à : aucune unité scientifique dans la mesure où les modèles théoriques développés restent étroitement liés à des disciplines et sous-disciplines qui, certes peuvent se croiser, mais n'en restent pas moins souveraines ; aucune unité technologique dans la mesure où les applications sont sans bornes prévisibles, car s'étendant aux domaines de l'électronique, des matériaux, du vivant et de la santé, de l'alimentation, du bâtiment, ... ; aucune unité industrielle puisque les nanotechnologies contribuent à toutes sortes de filières (la chimie, le nucléaire, l'électronique grand-public, les emballages, la métallurgie, les cosmétiques, ...) sans n'en fonder aucune.

Bien que des institutions scientifiques aient assuré la valorisation des nanosciences et des nanotechnologies ${ }^{17}$, cette promotion ne part pas des centres de recherche ou, dit autrement, n'est pas immédiatement le fruit de la science telle qu'elle se fait dans les laboratoires. Les nanosciences des années 2000 se présentent plutôt comme une suite, reformulée et réarticulée, de recherches menées au moins depuis les années 1980, amplifiées dans les années 1990. Le niveau nano apparaît d'ailleurs comme une « évidence » pour un chimiste habitué à travailler sur des molécules, un physicien confronté aux phénomènes quantiques ou un biologiste en prise avec les cellules. En conséquence, la promotion des nanosciences, donc de cet intitulé comme attraction scientifique, est indissociable du levier qu'ont constitué d'une part, le déplacement des politiques de la recherche vers la " preuve de concept » et la technologisation extensive de la science ${ }^{18}$, d'autre part, le changement du mode de financement de la recherche «scientifique », passant de la récurrence aux projets à durée déterminée. La politique nanotechnologique représente une opportunité d'obtention de financements, sur des sujets parfois simplement re-labellisés : «Avant, je faisais des couches minces, maintenant, je fais plus ou moins la même chose, mais ça s'appelle « nano $»^{19}$.

Le thème public des «nanos » constitue pourtant une inflexion dans la thématisation des risques technologiques. D'une part, du point de vue des enjeux : les nouveaux matériaux et, sur un autre plan, les nanobiotechnologies s'inscrivent dans une reconfiguration du mode d'industrialisation, reposant sur une différenciation technologique concurrentielle. D'autre part, du point

16 Séminaire inter-sciences Sonano ("Société et nanos "), Toulouse 2010-2011. Également : Joachim SCHUMMER, «On the Novelty of Nanotechnology : a Philosophical Essay », in Anthony Mark Cutter, Bert Gordijn (eds), In pursuit of NanoEthics, Springer, 2008, p. 15-30.

17 Rapport du groupe de travail « Nanotechnologies » de l'Académie des technologies, Les Nanotechnologies : enjeux et conditions de réussite d'un projet national de recherche, novembre 2002.

18 Donc le développement des technosciences et la réarticulation de la recherche fondamentale et appliquée (SCHUMMER, op. cit.). Voir également Bernard MIĖGE et Dominique VINCK, Les masques de la convergence Enquêtes sur sciences, industries et aménagements, Paris, Éditions des archives contemporaines, 2012.

19 Entretien avec un chercheur. 


\section{$\mathrm{C} \& O \mathrm{n}^{\circ} 45$}

de la pression opérée par l'espace public : les nanotechnologies s'inscrivent dans le processus de thématisation publique des risques, surprenant leurs promoteurs.

Dans un premier temps, en effet, le soutien aux " nanotechnologies » par l'administration américaine n'identifie pas de risques majeurs : "The government documents promoting nanotechnology that I have read make no mention of the risks of nanotechnology $y^{20}$. De même, en France, les premiers rapports institutionnels issus d'académies, des sciences ou des technologies, laissent de côté toute interrogation d'envergure sur les problèmes d'environnement et de santé que les nanotechnologies sont susceptibles de poser. Même si des chercheurs ${ }^{21}$ n'ignorent pas cette possibilité, comme en témoigne sa mise à l'ordre du jour, même marginale, lors d'un congrès " matériaux 2002 ", une «banalisation » des nanosciences par le milieu scientifique a pu favoriser leur découplage d'avec la problématisation des risques.

Il peut sembler paradoxal que les " nanotechnologies ", pourtant d'emblée tournées vers l'applicabilité, donc vers les produits, n'aient pas été spontanément accrochées par le système à la thématisation des risques environnement-santé, pourtant largement développée au cours des trois dernières décennies du $\mathrm{XX}^{\mathrm{e}}$ siècle. En effet, la question des risques associée aux produits a engendré, notamment, un " scandale » de l'amiante et une contestation virulente des OGM, qui ont fait reculer le système, au moins en Europe. Aux États-Unis, le poids des mouvements de consommateurs y est mesuré par de nombreuses class actions « réussies », sur des thèmes spécifiques (la consommation de tabac, par exemple, et même si, pour leur part, les OGM n'ont guère fait l'objet d'une contestation « advenue »).

En d'autres termes, au début des années 2000, tout se passe comme si les nanotechnologies étaient susceptibles d'effacer l'histoire des problèmes environnement-santé liés à l'industrie, à travers une expansion de technologies présentées comme étant nouvelles et avantageuses. De fait, la promotion de l'initiative nanotechnologique aux États-Unis s'appuie, non pas uniquement mais largement, sur la valorisation des possibilités de résoudre les problèmes d'environnement-santé. S’il est envisagé, par exemple, que le stockage de l'électricité, amélioré par le recours aux technologies nanométriques ${ }^{22}$, pourrait poser des questions sur le traitement et le recyclage des batteries, dans la balance entre les bénéfices et les risques le poids est explicitement situé du côté des bénéfices, et les risques du côté des conséquences inattendues : "Societal implications should be judged using a balanced approach between the

20 Bonnie NARDI, " A Cultural Ecology of Nanotechnology », in Mihail C. Roco and William Simms Bainbridge, Societal Implications of Nanoscience and Nanotechnology, March 2001, NSF, p. 248.

21 Entretien avec un chercheur.

22 Par l'inclusion de nano-objets aux propriétés améliorées en termes de conductivité. 
goals (leading to envisioned societal benefits) and unexpected consequences (which could be a combination of unexpected benefits and risks) ${ }^{23}$.

\section{... à l'implicabilité sociétale}

De façon relativement inédite, l'annonce d'une grande politique scientificotechnique des nanotechnologies s'est quasi-immédiatement accompagnée de l'émergence de sa contestation civique, s'étalant de l'Australie et le Canada vers d'autres pays, dont la France. Cette contestation relève d'une multiplicité d'approches. Trois thèmes fondamentaux se construisent dans l'espace public, à propos de : la miniaturisation électronique, susceptible de renforcer le développement de dispositifs de surveillance de la vie publique ou privée ; la convergence NBIC (Nanotechnologies, Biotechnologies, Information, Cognition) questionnant la notion même de « vivant » à travers la capacité d'engendrer un " humain augmenté »; les risques potentiels pour l'environnement-santé, essentiellement attribués au développement des nanomatériaux.

Bien que ces problèmes aient antérieurement été l'objet de mises en public particularisées dans la tradition contestataire, l'unification opérée par la politique nanotechnologique a eu pour effet de faire converger des contestations jusqu'alors partielles ${ }^{24}$.

En ce sens, les trois thèmes - droits fondamentaux, vivant et environnementsanté - se consolident et s'agrègent les uns aux autres, engendrant une tendance à élargir le fondement de ce qui constitue le ressort de la contestation : l'implicabilité, de grande ampleur, de l'innovation nanotechnologique.

Ce passage rapide des applications possibles aux implications effectives traverse les pratiques des centres de recherche, dont certains trouvent une sorte d'exutoire à travers l'idée d'une réduction ou minimisation des risques dès la conception via le «safe(r) by design $n^{25}$ ». Néanmoins, cette «solution » n’inverse pas la tendance à la thématisation publique des nanotechnologies, en particulier à travers la formulation de revendications de plus en plus précises sur les risques environnement-santé.

Cette démarche contestataire ne s'appuie pas, à la différence d'autres thèmes publics de risques, sur une identification avérée des dangers intrinsèques aux substances et produits concernés. Pour sa part, dès les années 1960, la mise en thème du nucléaire civil s'était nourrie de la connaissance des effets de la radioactivité et de l'expérience des explosions militaires au Japon. La critique du bien-fondé des activités industrielles " classiques " s'est constamment

23 Mihail C. ROCO, « Broader societal issues of nanotechnology ", Journal of Nanoparticle Research 5: 181189,2003 , p. 184. Ou encore : « To assess a nanotechnology (or any technology) in terms of its unintended consequences, researchers must examine the entire system of which the technology is a part through its entire life cycle. ", Roco and Bainbridge, op. cit., p. 11.

24 Marie-Gabrielle SURAUD, Les nanoactivités à l'épreuve de leur légitimation. Rapport pour le volet sociétal de Nano Innov et le programme REPERE du ministère de l'Écologie. Certop, Toulouse, 2011,

25 Christopher KELTY, op. cit. 
alimentée : d'une connaissance des potentiels de catastrophes, dont certaines ont scandé l'industrialisation (marées noires, accidents industriels, ...), du constat des pollutions chroniques et de l'accélération du réchauffement climatique, ou encore des conséquences toxiques, lentement reconnues, de minéraux telles que l'amiante ou le charbon.

Dans le cas des nanotechnologies, la mise en thème public des risques se développe sans ou avant que n'aient été formellement ou techniquement identifiés des dangers, c'est-à-dire des comportements toxiques et écotoxiques spécifiques aux substances concernées. En ce sens, le thème des nanotechnologies prolonge et amplifie celui des OGM, marqué par le même type d'inconnu, mais dont l'applicabilité est délimitée, à la différence de celle des nanotechnologies.

Cette identification des dangers potentiels des nanotechnologies apparaît particulièrement problématique en raison de la multiplicité des familles d'objets : nanocarbones, nanoargents, nanodioxydes de titane, ..., et de la démultiplication des objets eux-mêmes : deux nanotubes de carbone ne sont a priori pas identiques quand ils sortent d'ateliers différents, voire quand diffèrent les catalyseurs utilisés pour les synthétiser. À cela s'ajoute l'abondance des liaisons - fortes ou faibles - d'une substance avec d'autres substances, nanométriques ou non, conduisant à des comportements toxiques ou écotoxiques variables selon les liaisons. Le nombre incommensurable de situations possibles obère, de facto, la possibilité de tirer des conclusions établies quant aux risques environnement-santé des produits concernés.

Cette lacune, qui n'est pas sur le point d'être palliée ${ }^{26}$, a favorisé une radicalisation de la contestation des "nanos », alertée par l'étendue des applications techno-industrielles annoncées ou envisageables. Ainsi observe-t-on une démultiplication de demandes de moratoire relatives aux nanotechnologies : la Fédération des industries chimiques de la CGT se prononce en faveur d'un moratoire sur les produits issus des nanotechnologies; les Amis de la Terre (France) pour un moratoire général sur toute recherche concernant les «nanos »; d'autres associations pour des moratoires sélectifs, sur la recherche appliquée et la commercialisation des nanoproduits (Fondation Sciences Citoyennes) ou sur les produits non-médicaux en contact avec la peau (France Nature Environnement).

Ces demandes se sont progressivement formalisées à travers des discussions publiques / civiques, spontanées ou construites, à l'échelle européenne ou française, dans le sillage de l'adoption du règlement européen Reach (Registration, Evaluation, Authorization of CHemical Substances) et de son principe central : "pas de données [toxicologiques ou écotoxicologiques], pas de marché».

26 Cette assertion s'appuie sur la participation (observante) à un groupe de travail au sein d'une agence publique d'expertise et de nombreux entretiens, formels ou informels. 
La thématisation rapide de l'implicabilité sociétale croissante de l'innovation nanotechnologique est ainsi la marque d'une prégnance de plus en plus forte de l'espace public dans le processus de légitimation des activités concernées, ce qu'on peut traduire comme étant la mise en œuvre d'un « processus de précaution ".

\section{... jusqu'au processus de précaution}

On le sait $^{27}$, le principe de précaution s'est d'abord manifesté, en République Fédérale d'Allemagne au cours des années 1960, à travers une orientation générale destinée à encadrer les politiques de développement durable $^{28}$. Puis il s'est formalisé, juridiquement, en Europe, mais aussi en France au milieu des années 1990, aboutissant à son inclusion dans la Charte constitutionnelle de l'environnement de 2005 (article 5) à la suite d'années de débats, périodiquement relancés ${ }^{29}$, sur la pertinence du principe et de sa formulation concrète.

Considéré de façon théorique, le principe de précaution reflète une tension entre, d'une part, des exigences universalisables : l'adoption de mesures effectives et proportionnées afin de prévenir un risque de dommages graves et irréversibles à l'environnement, et, d'autre part, le calcul monétaire : le principe s'adapte « à un coût économiquement acceptable ».

Pour sa part, la notion de " processus de précaution ", avancée dans le présent texte, traduit non pas tant une règle instituée qu'une dynamique sociale singulière, non réductible au poids de l'incertitude scientifique, résultant des difficultés des « sciences » à établir ou valider l'existence d'un danger. Ces difficultés ne peuvent être déconnectées du fait que des disciplines comme la toxicologie et ou l'écotoxicologie, ne sont, à ce stade, que faiblement prédictives du danger associé à un objet ayant des caractéristiques physicochimiques particulières. En conséquence, l'identification du danger d'un matériau nouveau dépend encore largement de la construction de données épidémiologiques, donc a posteriori, sur une période d'une dizaine d'années au moins, voire beaucoup plus.

Cependant, s'il y a bien une incertitude scientifique - durable - quant aux dangers potentiels des nanoproduits, elle n'explique pas la formation du processus de précaution, au sens où ce dernier n'a pas l'incertitude pour ressort. Par analogie négative, dans d'autres circonstances l'absence (rémanente) de solutions connues pour les déchets nucléaires / radioactifs n'avait pas entravé l'expansion des centrales nucléaires au cours des années 1960-1970.

27 Dominique BOURG et Kerry H. WHITESIDE, «Précaution : un principe problématique mais nécessaire », Le Débat, Gallimard, 2004/2 - n 129, p. 153 à 174.

28 Nicole D’ALMEIDA, « Le développement durable entre temps court et temps long, entre principe de gestion et principe de discussion », Communication et organisation, 29 | 2006, URL : http://communicationorganisation. revues.org/3393.

29 Notamment par l'Office Parlementaire des Choix Scientifiques et Techniques (OPCST). 
Par conséquent, considérée du point de la problématisation des risques, l'importance acquise par l'incertitude scientifique dans les controverses relève d'une construction politique, c'est-à-dire de la mise en thème public des risques et de l'embarras du système pour faire valoir ses " solutions ». C'est le dépliement du processus de précaution, donc de l'exigence sine qua non issue de l'espace public que le bien-fondé d'innovations à risques soit légitimé, qui a transformé la question de l'incertitude scientifique en une épreuve à surmonter par la sphère de la décision fonctionnelle. L'impossibilité de démontrer l'absence de risques associés aux substances nanométriques et, conformément au règlement européen Reach, la nécessité pour les entreprises de prouver leur maîtrise écologique de l'utilisation de ces substances, ont déplacé les principes de la légitimation, en faisant de l'incertitude scientifique une composante de la thématique publique des risques.

Cette publicisation des limites de la capacité à justifier des décisions, tant politico-bureaucratiques qu'économiques, s'avère d'autant plus problématique que la dynamique de l'espace public s'est élargie. En effet, le thème des nanotechnologies met en évidence une conjonction des revendications sur le travail, l'environnement et la consommation autour de la précaution ${ }^{30}$.

À la différence de thématisations antérieures confrontant (nucléaire, risques de catastrophe industrielle, amiante) ou séparant sans conflit particulier (OGM) l'espace du travail salarié à l'espace public, le thème des nanotechnologies a un effet unificateur. Le syndicalisme a ainsi privilégié ses relations avec la sphère associative plutôt qu'avec les employeurs ${ }^{31}$.

Ce thème est en effet marqué par un décloisonnement des problèmes de santé au travail, longtemps confiné à l'espace des relations professionnelles ${ }^{32}$, et par un décentrage des revendications syndicales vis-à-vis du calcul économique, dépassant largement la critique du mode de fonctionnement tendu des organisations productives. En conditionnant, même de manière variable, le développement des nanotechnologies au traitement préalable des risques pour le travail aussi bien que pour l'environnement (et la santé en général), la convergence des revendications sur le travail et sur l'environnement-santé manifeste la prégnance croissante de l'espace public et des exigences universalisables qui en sont issues.

Cette prégnance se traduit concrètement par le repli observable de la valorisation des nanotechnologies ${ }^{33}$ et les difficultés économiques rencontrées par des producteurs. L'objet le plus controversé - le nanotube de carbone - se voit interrogé par le système lui-même comme en témoignent, en France,

30 Patrick CHASKIEL, «Syndicalisme et Nanotechnologies. De l'espace des relations professionnelles à l'espace public des risques ", Sociologie du travail, n’ 55/4, 2013, p. 554-574.

31 Entretien avec un secrétaire général-adjoint de la Confédération Européenne des Syndicats.

32 Emmanuel HENRY, "Politiques de santé au travail : une difficile mise en débat public », Santé et Travail, septembre 2009, $\mathrm{n}^{\circ} 068$.

33 Cette assertion résulte d'expériences partagées de recherche menées sur le terrain et d'entretiens avec des agents d'institutions administratives de contrôle des risques. 
sa commercialisation problématique ${ }^{34}$ et, en Allemagne, la fermeture d'un atelier spécialisé très peu de temps après son lancement ${ }^{35}$.

L'exigence conjointe, formulée par le syndicalisme européen et neuf $\mathrm{ONG}^{36}$, d'une renégociation spécifique du règlement $R$ each, afin de le rendre applicable aux nanotechnologies, exprime la formation renouvelée d'une thématique des risques technologiques, portée à l'échelle des institutions de l'Union Européenne.

\section{Conclusion : vers un « désenchantement " de l'industrialisation ?}

En se constituant publiquement / civiquement, la question des nanotechnologies a infléchi le mode de prise de décisions, industrielles et étatiques. Elle a accéléré la formation d'un processus de précaution, c'est-àdire d'une « mise sous conditions » du développement des nanotechnologies.

Cette dynamique publique ouvre une perspective relativement nouvelle vis-à-vis des préoccupations de la théorie habermassienne.

Dans sa partition des opérations sociales entre régulation et communication, générant une différenciation entre système et monde vécu, Habermas avait laissé peu de place au « conflit de classes », jugé pacifié37. Le travail salarié ne lui apparaissait plus comme l'expérience primordiale de l'oppression, à côté de la montée des problèmes tels que ceux relatifs aux usages privés du corps ou à la protection de l'environnement-santé.

Or, le thème des nanotechnologies tend à montrer que, en se rapportant à l'espace public, les revendications sur le travail deviennent des exigences posées en amont de l'industrialisation et se détachent d'une pensée, non unique, mais dirigeante au sein de la classe ouvrière, qui a fait du " progrès technologique » le moteur du progrès social. Du coup, la question des risques au travail se présente comme une source de conflits, susceptible de renouveler les rapports entre salariés et patronat, de par une pression accrue de l'espace public, notamment européen, sur le système économique : la seule perspective d'emplois (potentiellement) à venir ne suffit plus à rendre une décision ipso facto légitime.

Par voie de conséquence, l'expansion du système économique, plus particulièrement de l'industrie, se heurte à une limitation. Alors que l'expansion industrielle pouvait s'appuyer sur une base sociale - le mouvement ouvrier historique - une tendance au « désenchantement »(Weber) de l'industrie est bien installée dans le monde du travail salarié, mobilisant des énergies dépassant la seule dénonciation des « dégâts du progrès » par la CFDT. Ce désenchantement engendre une réduction de la maîtrise du système sur

34 Entretiens multiples.

$35 \mathrm{http} / /$ www.press.bayer.com/baynews/baynews.nsf/id/Bayer-MaterialScience-brings-nano-projects-to-a-close 36 Déclaration du 23 octobre 2012.

37 Jürgen HABERMAS, Théorie de l'agir communicationnel. Pour une critique de la raison fonctionnaliste (t. 2), Paris, Fayard, 1987, p. 367. 


\section{$\mathrm{C} \& O \mathrm{n}^{\circ} 45$}

les conditions de son expansion. Même si ce dernier peut régulièrement monétariser des activités correspondant aux exigences de l'espace public (les énergies renouvelables, par exemple), la seule dimension économique suffit de moins en moins à justifier les décisions fonctionnelles. La complexité introduite par les besoins renouvelés en légitimité des décisions crée de l'incertitude quant à la réalisation des opérations projetées par le système. Ainsi ce dernier est-il devenu très sensible à l'incertitude écologique susceptible de peser tout au long de la chaîne de création de la valeur économique.

Cette incertitude écologique produite par l'espace public implique de s'affranchir de réflexions privilégiant, au fond, la puissance du système. Elle incite à adopter une approche dans laquelle il est attribué de la puissance à l'agir communicationnel, pour examiner les issues de la confrontation entre système et monde vécu.

\section{BIBLIOGRAPHIE}

BOLTANSKI Luc, Chiapello Eve, Le nouvel esprit du capitalisme, Paris, éd. Gallimard, 1999

BOURDIEU Pierre, La distinction. Critique sociale du jugement, Paris, éd. de Minuit, 1979 ,

BOURG Dominique Whiteside Kerry H., « Précaution : un principe problématique mais nécessaire », Le Débat, Gallimard, 2004/2 - n 129, p. 153 à 174.

CHASKIEL Patrick, « Syndicalisme et Nanotechnologies. De l'espace des relations professionnelles à l'espace public des risques ", Sociologie du travail, n ${ }^{\circ}$ 55/4, 2013, p. 554574.

D'ALMEIDA Nicole, « Le développement durable entre temps court et temps long, entre principe de gestion et principe de discussion ", Communication et organisation, 29 | 2006, URL : http://communicationorganisation.revues.org/3393.

HABERMAS Jürgen, L'espace public. Archéologie de la publicité comme dimension constitutive de la société bourgeoise, Paris, Payot, 1978, 294 pages.

- Théorie de l'agir communicationnel. Pour une critique de la raison fonctionnaliste (t. 2), Paris, Fayard, 1987, 480 pages.

- Droit et Démocratie. Entre faits et normes, Paris, Gallimard, 1997, 560 pages

KELTY Christopher M., "Beyond Implications and Applications: the Story of "Safety by Design" ", NanoEthics 3, no. 2, 2009, 18 pages.

LUHMANN Niklas, Ecological Communication, Chicago, The University of Chicago Press, 1986, 187 pages

- Theory of Society, volume 1, Stanford, Standford University Press, 2012, 453 pages

MIĖGE Bernard et VINCK Dominique, Les masques de la convergence Enquêtes sur sciences, industries et aménagements, Paris, Éditions des archives contemporaines, 2012, 396 pages. 
NARDI Bonnie, "A Cultural Ecology of Nanotechnology », in Mihail C. Roco and William Simms Bainbridge, Societal Implications of Nanoscience and Nanotechnology, March 2001, NSF, 6 pages.

Rapport du groupe de travail « Nanotechnologies » de l'Académie des technologies, Les Nanotechnologies : enjeux et conditions de réussite d'un projet national de recherche, novembre 2002, 54 pages

Rapport : «Le déploiement industriel des nanotechnologies et de la biologie de synthèse sur les territoires, précurseur des manufactures du futur ", rapport au gouvernement, N 13-1207 DEF/CGARM/SG, décembre 2013, 153 pages.

ROCO Mihail C. and BAINBRIDGE William Simms, Societal Implications of Nanoscience and Nanotechnology, March 2001, NSF, 280 pages.

ROCO Mihail C., "Broader societal issues of nanotechnology ", Journal of Nanoparticle Research 5: 181-189, 2003, 9 pages.

- NNI: From Vision to the Implementation. Brief presentation, November 2000, https:/www.nsf.gov/crssprgm/nano/reports/roco_vision.jsp.

SCHUMMER Joachim, "On the Novelty of Nanotechnology: a Philosophical Essay ", in Anthony Mark Cutter, Bert Gordijn (eds), In pursuit of NanoEthics, springer 2008, http://www.joachimschummer.net/papers/2008_Novelty-of-Nanotechnology_ Cutter-Gordijn.pdf, 15 pages.

SURAUD Marie-Gabrielle, et al., Les nanoactivités à l'épreuve de leur légitimation. Rapport pour le volet sociétal de Nano Innov et le programme REPERE du ministère de l'Écologie. Certop, Toulouse, 2011, 46 pages.

VINCK Dominique, Nano-technologies, Paris, Le Cavalier bleu, 2009, 128 pages.

Résumé : Alors que bon nombre de recherches s'inspirant, notamment, d'Habermas partent du principe de domination, c'est-à-dire de l'idée d'une perte de contrôle des citoyens sur l'expansion des activités économiques et politiques, le présent texte, en s'appuyant sur le thème des nanotechnologies, montre en quoi l'agir communicationnel est capable de limiter et contrôler significativement cette expansion.

Mots-clés : nanotechnologies, espace public, risques, précaution, contestation, industrie.

Abstract : Though many researches notably following Habermassian theory, deal with societal relations through a domination approach, i.e. adopt the very idea that citizens have a weaker and weaker grip on economic and political activities, this text based on a study of nanotechnologies theme, shows how communicative action is able to significantly limit and control this expansion.

Keywords : nanotechnologies, public sphere, risks, precaution, protests, industries. 
